This is the Author's Accepted Manuscript version.

This is the peer reviewed version of the following article: Maniruzzaman, $M$. and Douroumis, D. (2014), An in-vitro-in-vivo taste assessment of bitter drug: comparative electronic tongues study. Journal of Pharmacy and Pharmacology. doi: 10.1111/jphp.12319, which has been published in final form at http://dx.doi.org/10.1111/iphp.12319. 


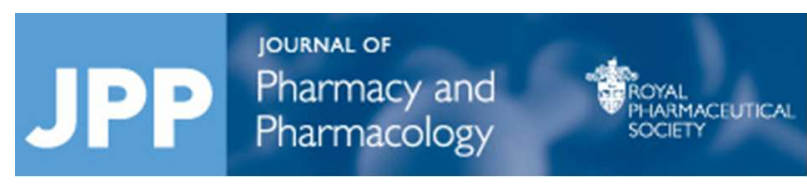

\section{An in vitro-in vivo taste assessment of bitter drug: Comparative electronic tongues study}

\begin{tabular}{|c|c|}
\hline Journal: & Journal of Pharmacy and Pharmacology \\
\hline Manuscript ID: & JPP-14-0230.R1 \\
\hline Wiley - Manuscript type: & Research Paper \\
\hline Date Submitted by the Author: & $\mathrm{n} / \mathrm{a}$ \\
\hline Complete List of Authors: & $\begin{array}{l}\text { Maniruzzaman, Mohammed; University of Greenwich, School of Science } \\
\text { Douroumis, Dennis; University of Greenwich, Medway School of Science }\end{array}$ \\
\hline Keywords: & $\begin{array}{l}\text { In vivo/in vitro Correlation < Biopharmaceutics and Drug Disposition, } \\
\text { Pharmaceutical Analysis, Pharmaceutics and Drug Delivery }\end{array}$ \\
\hline Abstract: & $\begin{array}{l}\text { Objectives The efficiency of the Astree e-tongue and Taste Sensing system } \\
\text { TS5000Z for the evaluation of the taste masking effect of hot melt } \\
\text { extruded formulations was investigated in this study. } \\
\text { Methods Hot melt extrusion (HME) processing was optimized using } \\
\text { Randcastle single screw extruder (USA) to manufacture extrudates with } \\
\text { desirable characteristics. Cationic model drug propranolol HCl (PRP) was } \\
\text { processed with the anionic polymers - Eudragit L100ß (L100) and Eudragit } \\
\text { L100-55 (Acryl-EZE). In vitro taste masking efficiency of the two polymers } \\
\text { was performed by using two different e-tongues (Astree e-tonge and } \\
\text { TS5000Z). } \\
\text { Key Findings Both e-tongues were able to detect the taste masking } \\
\text { variations of the extrudates and were in good agreement with the in vivo } \\
\text { results obtained from a panel of six healthy human volunteers (R2>0.84). } \\
\text { However, each e-tongue sensor demonstrated different sensitivity } \\
\text { suggesting a careful consideration of the experimental findings during melt } \\
\text { extrusion is necessary for the development of taste masked formulations. } \\
\text { Furthermore, FT-IR spectroscopy and NMR studies revealed possible drug } \\
\text { polymer intermolecular interactions as the mechanism of successful taste } \\
\text { masking. } \\
\text { Conclusions HME can effectively be used to manufacture taste masked } \\
\text { extruded formulations while both e-tongues demonstrated satisfactory } \\
\text { taste analysis for the development of taste masked formulations. }\end{array}$ \\
\hline
\end{tabular}




\title{
An in vitro-in vivo taste assessment of bitter drug: Comparative electronic tongues study
}

\author{
Mohammed Maniruzzaman $^{1 *}$ and Dennis Douroumis ${ }^{1}$
}

${ }^{1}$ School of Science, University of Greenwich, Central avenue, Chatham Maritime, Chatham, Kent, ME4 4TB, UK

\footnotetext{
* To whom correspondence should be addressed: Mohammed Maniruzzaman, University of Greenwich, Medway School of Science, Chatham Maritime, ME4 4TB, Kent, UK, email: M.Maniruzzaman@gre.ac.uk. Phone: 0044(0)208 331 8342, Fax: 0044 (0) 2083319805.
} 


\section{Abstract}

3 Objectives The efficiency of the Astree e-tongue and Taste Sensing system TS5000Z for the 4 evaluation of the taste masking effect of hot melt extruded formulations was investigated in 5 this study.

6 Methods Hot melt extrusion (HME) processing was optimized using Randcastle single screw 7 extruder (USA) to manufacture extrudates with desirable characteristics. Cationic model drug 8 propranolol $\mathrm{HCl}(\mathrm{PRP})$ was processed with the anionic polymers - Eudragit L100® (L100) 9 and Eudragit L100-55 (Acryl-EZE). Solid state of the drug in polymer matrices was 10 evaluated by scanning electron microscopy (SEM), differential scanning calorimetry (DSC), 11 particle size analysis, Fourier Transform Infra-Red (FT-IR) and Nuclear Magnetic Resonance 12 (NMR) analysis. In vitro taste masking efficiency of the two polymers was performed by 13 using two different e-tongues (Astree e-tonge and TS5000Z). The results obtained from both 14 e-tongues were further compared and contrast to find out the sensor outputs in all 15 formulations.

16 Key Findings Solid state analysis of the extruded formulations revealed the presence of 17 amorphous PRP. Both e-tongues were able to detect the taste masking variations of the 18 extrudates and were in good agreement with the in vivo results obtained from a panel of six 19 healthy human volunteers $\left(\mathrm{R}^{2}>0.84\right)$. However, each e-tongue sensor demonstrated different sensitivity suggesting a careful consideration of the experimental findings during melt

21 extrusion is necessary for the development of taste masked formulations. Furthermore, FT-IR 22 spectroscopy and NMR studies revealed possible drug polymer intermolecular interactions as 23 the mechanism of successful taste masking.

24 Conclusions HME can effectively be used to manufacture taste masked extruded 25 formulations while both e-tongues demonstrated satisfactory taste analysis for the 26 development of taste masked formulations.

27 Keywords: Taste masking, Propranolol HCl®, Eudragit L100®, Acryl-EZE, Astree E28 Tongue, TS5000Z. 


\section{Introduction}

Masking the bitter taste of active pharmaceutical ingredients (APIs) is considered a major challenge especially for the development of orally administered dosage forms in pharmaceutical industry. ${ }^{[1,2]}$ Due to the unpleasant sensation bitter taste is always the least desired and sometimes completely undesired option whereas sweet taste is pleasant for most of the people regardless their age and origin. ${ }^{[3]}$ In reality most of the APIs used in oral drug products have a bitter taste which is not only undesirable but also frequently has a negative influence on the palatability of the final dosage forms. For paediatric population unpalatable/bitter taste is the most challenging as children are highly sensitive to taste and actively refuse the unpalatable drugs. ${ }^{[4,5]}$ It is often impossible to block bitter taste receptors (due to their increased numbers) from the drug without compromising the mechanism of its action. ${ }^{[6,7]}$ The extent of taste masking of an API depends almost exclusively on the type of formulation (solid or liquid). Being the first preference, commercial oral liquid dosage forms contain artificial sweeteners (e.g saccharin and aspartame) and flavours to mask the tastes which are often limited due to the regulatory requirements. Due to very poor effects of this method and possibilities of toxic and allergic reactions, European Medicines Agency (EMA) strongly recommends another way for taste masking instead of adding sweeteners or flavours. [8] An ideal solution for this problem should involve the prevention of any contact of the unpalatable APIs with the taste buds without the addition of taste additives. Such an ideal formulation can be developed by applying an appropriate coating or encapsulation on the API encapsulated drug then can be dispersed in water.

In vivo taste masking evaluation studies are performed by healthy human volunteers and involve taste assessments based on the individual scores. ${ }^{[11]}$ A well-established statistical method is required to overcome errors and variability between volunteers within the limit of threshold taste perceptions. According to the FDA guidelines studies on paediatric formulations should not be performed on paediatric volunteers due to ethical conflicts. On the other hand in order to design paediatric formulations mature volunteers should also be prohibited due to large physiological differences of taste sensation. ${ }^{[7]}$ Ethical doubts on experiments in children and difficulties with interpretation of the results eventually indicate the need to use alternative in vitro methods for taste evaluations. ${ }^{[4,7]}$ In the last few years, electronic tongues (e-tongues) became popular for the evaluation of the in vitro taste performance for repeatable analysis of pharmaceutical products. ${ }^{[12,13]}$ Electronic tongues are sensor array systems which are able to determine single substances as well as complex mixtures of various substances. Electronic -tongue is a device simulating human sense of 
taste that allows for the identification and classification of liquid samples. Regardless of the selected chemical compounds e-tongues work to the recognition of general sample properties such as specific taste (e.g bitter). They consist of an array of chemical sensors and a pattern recognition system. ${ }^{[14-16]}$ Over the last few years electronic tongue systems have taken the advantage of different measuring principles including potentiommetry, voltammetry and amperometry. Currently, there are commercially available e-tongues which have successfully been employed for taste assessments in various pharmaceutical formulations. Astree e-tongue (Alpha MOS, France) and taste sensing system TS-5000Z (INSENT, Japan) are two common e-tongues which have been used as in vitro taste assessment tools

The aim of this novel study is the evaluations of the taste masking efficiency of hot melt extruded formulations of bitter API (PRP) by using two different e-tongues (Astree etongue and TS-5000Z) simultaneously and studying the mechanism of the effective taste masking via extrusion processing.

\section{Materials and methods}

\section{Materials}

Propranolol $\mathrm{HCl}$ (PRP) was purchased from Sigma Aldrich (London, UK). Eudragit L100 (L100) and Eudragit L100-55 (Acryl-EZE) was kindly donated by Evonik Pharma Polymers (Darmstadt, Germany) and Colorcon ltd respectively. The HPLC solvents were of analytical grade and purchased from Fisher Chemicals (UK). All materials were used as received.

\section{Preparation of formulation blends and hot-melt extrusion (HME) processing}

PRP formulations with L100 and Acryl-EZE to be extruded were mixed properly in $100 \mathrm{~g}$ batches for $10 \mathrm{~min}$ each. A Turbula (TF2, Basel) mixer was used to blend the powder formulations (drug/polymers ratio used were 10:90 w/w). Extrusion of all PRP formulations were performed using a Randcastle single-screw extruder (RCP 0625, USA) equipped with a $5 \mathrm{~mm}$ rod die using $100^{\circ} \mathrm{C} / 113^{\circ} \mathrm{C} / 155^{\circ} \mathrm{C} / 155^{\circ} \mathrm{C} / 155^{\circ} \mathrm{C}$ (Feeder to die) temperature profiles. The screw speed maintained for all extrusion was $15 \mathrm{rpm}$. The produced extrudates (strands) was grinded by using a Ball Milling system ( 8 balls, $1.5 \mathrm{~cm}$ diameter) to obtain granules $(<500 \mu \mathrm{m})$. Grinding by ball milling was carried out with a rotational speed of $400 \mathrm{rpm}$ for 5 $\min$.

\section{Particle morphology and size distribution}

Scanning Electron Microscopy (SEM) was used to study the surface morphology of the extrudates. Samples were mounted on an aluminum stage using adhesive carbon tape which was then placed in a low humidity chamber prior to the analysis. Samples were also coated 
with gold-palladium, and microscopy was performed using Cambridge Instruments - S630 (Cambridge, UK) operating at an accelerating voltage of $5 \mathrm{kV}$. All samples were run singlet.

The particle size distribution of the micronized granules of all formulations was measured by dry sieving. The method involved stacking of the sieves on top of each other and then placing the test powder (50 g) on the top sieve. The nest of sieves was subjected to a standardized period of agitation (20 min) and then the weight of the material retained on each sieve was accurately determined to give the weigh percentage of powder in each sieve size range. All samples were run triplicate.

\section{Differential scanning calorimetry (DSC) and Modulated temperature DSC analysis}

DSC runs of pure actives, physical mixtures and extrudates were carried out using a MettlerToledo 823e (Greifensee, Switzerland) differential scanning calorimeter. Sealed aluminium pans were used to prepared sample weighing about 2-5 mg of samples $(n=3)$. Samples were heated at $10^{\circ} \mathrm{C} / \mathrm{min}$ heating rate from -40 to $220^{\circ} \mathrm{C}$. At the time of heating, samples were under nitrogen atmosphere. In addition modulated temperature differential scanning calorimetry (MTDSC) studies were performed from $25^{\circ} \mathrm{C}$ to $250^{\circ} \mathrm{C}$ with an underlying heating rate of $1^{\circ} \mathrm{C} / \mathrm{min}$. The pulse height was adjusted to $1-2^{\circ} \mathrm{C}$ with a temperature pulse width of $15-30 \mathrm{~s}$.

\section{In vivo taste masking evaluation}

In vivo taste masking evaluation of pure API, polymers and all active extruded formulations was performed in accordance to the Code of Ethics of the World Medical Association (Declaration of Helsinki). ${ }^{[19]}$ Six (6) healthy volunteers of either sex (age 18-25) were selected $($ Male $=3$, female $=3$ ) from whom informed consent was first obtained (approved by the Ethics Committee of the University of Greenwich, Ref: UG09/10.5.5.12). All volunteers were also trained prior to the experiment. The equivalent of $100 \mathrm{mg}$ of pure PRP or PRP based extrudates (containing equal amounts of API) were held in the mouth for 60 seconds and then spat out. The selection of samples was random and in between of two samples analysis mineral water was used to wash each volunteer's mouth. The bitterness was recorded immediately according to the bitterness intensity scale from 1 to 5 where 1, 2, 3, 4 and 5 indicate none, threshold, moderate, bitter and strong bitterness. All samples were evaluated in triplicate.

\section{In vitro taste masking evaluation: Astree E-Tongue (Alpha MOS)}

The assays were better performed on Astree e-tongue system equipped with an Alpha M.O.S. sensor set \#2 (for pharmaceutical analysis) composed of 7 specific sensors (ZZ, AB, BA, BB, CA, DA, JE) on a 48-positions autosampler using $25 \mathrm{ml}$ beakers. Acquisition times were 
132 fixed at 120s. [19] All the data generated on Astree system were treated using

133 multidimensional statistics on AlphaSoft V12.3 software. Each solution was tested on Astree

134 e-tongue at least 3 times. 3 replicates were taken into account for the statistical treatment. The

135 average values of all sensors signals between 100 and $120 \mathrm{~s}$ constitute the raw data for later

136 multivariate statistical data processing. This processing allows to map the data on 2-

137 dimension maps (Principal Components Analysis - PCA, Discriminant Factorial Analysis,

138 Statistical Quality Control, Partial Least Square, etc). With Astree e-tongue, PCA was used

139 to evaluate the differences and similarities between various samples or groups of samples.

140 The samples are represented in a two-dimensional or three-dimensional space with reference

141 to the selected components (PC1 and PCn). The components are classified according to the

142 level of information they produce. Astree sensors were cleaned up with deionised water

143 between each sample measurement.

\section{Sample preparation for Astree E-Tongue}

145 In vitro taste masking evaluation was carried out with an Astree E-Tongue equipped with 7

146 different sensor sets. To be as close as panellists taste's conditions, each drug was diluted for $14760 \mathrm{~s}$ under magnetic stirring in $25 \mathrm{ml}$ of deionised water to reach API concentration 148 corresponding to a final dose of $100 \mathrm{mg}$. Then solutions were filtered (as the particles can 149 damage the ASTREE sensors and thus alter the quality of results) with Buchner funnel fitted 150 with filter paper at $2.5 \mu \mathrm{m}$ pore size (Table 1). Analysis for each API was done in triplicate.

\section{In vitro taste masking evaluation: $\mathrm{TS}-5000 \mathrm{Z}$ sensing system}

152 The assays were realized on TS-5000Z taste sensing system equipped with a BASIC sensor 153 set (for pharmaceutical analysis) which are suitable for basic APIs composed of 10 specific 154 sensors (AAE, CT0, CA0, C00, AE1, AC0, AN0, BT0, GL1 ) on a 48-positions autosampler 155 using $25 \mathrm{ml}$ beakers. Each measurement cycle was consisted of measuring a reference 156 solution ( $\mathrm{Vr}$ ) followed by sample solution (Vs) and then the aftertaste (Vr) followed by a 157 cleaning procedure. The "aftertaste" was measured by determining the change in membrane 158 potential caused by the adsorption of the analyte to the lipid membrane. Sensor outputs for 159 both taste (also called relative value $(\mathrm{R})$ ) and "aftertaste" were then calculated in relation to 160 the initially determined sensor response to the reference solution ( $\mathrm{Vr}){ }^{[1,22]}$ Acquisition times 161 were fixed at 120 s with a BT0 negatively charged sensor. All the data generated on TS$1625000 \mathrm{Z}$ system were treated using multidimensional statistics. Each solution was tested on TS$1635000 \mathrm{Z}$ at least 4 times and triplicates were taken into account for the statistical treatment. 164 Sensors were then cleaned up in references solutions $(30 \mathrm{mM} \mathrm{KCl}+0.3 \mathrm{mM}$ tartaric acid) 165 between each sample measurement. The samples were dissolved in $50 \mathrm{~mL}$ of $10 \mathrm{mM} \mathrm{KCl} \mathrm{aq.}$ 
166 solutions and further diluted to prepare $0.03,0.1,0.3$, and $1 \mathrm{mM}$ solutions as standards. Then

167 solutions were filtered with Buchner funnel fitted with filter paper at $2.5 \mu \mathrm{m}$ pore size $(\mathrm{n}=3)$.

168 FTIR spectroscopy

169 FT-IR analysis was performed on the drug, polymer, drug/polymer physical mixtures, and 170 extrudates using Perkin Elmer PE1600 (Massachusetts 02451

171 USA) Fourier Transform Infrared Spectra according to the KBr disc method from $400-3600$ 172 wavelength $/ \mathrm{cm}^{-1}$ range $(\mathrm{n}=3)$.

173 Nuclear magnetic resonance (NMR) studies

174 NMR spectra were recorded on a Jeol ECA 500 NMR spectrometer, incorporating a $5 \mathrm{~mm}$ 175 inverse probe (The ${ }^{1} \mathrm{H}$ operating frequency was $500 \mathrm{MHz}$ ). ${ }^{1} \mathrm{H}$ NMR spectra of the drugs, 176 polymers and drug/polymer formulations were recorded using the standard Jeol pulse 177 sequence. All samples were dissolved in $\mathrm{CD}_{3} \mathrm{OD}$, degassed and then maintained at $25^{\circ} \mathrm{C}$ 178 during data acquisition. Samples were referenced with respect to the solvent. The solution 179 concentration of the drug was $2 \mathrm{mg} / \mathrm{ml}$, the polymers were $18 \mathrm{mg} / \mathrm{ml}$, and the drug/polymer 180 formulation was $20 \mathrm{mg} / \mathrm{ml}$ (the overall drug content in the formulations was $10 \%)(\mathrm{n}=3) .{ }^{1} \mathrm{H}$ $181 \mathrm{~T}_{1}$ relaxation experiments were recorded for all samples using a standard inverse recovery 182 experiment. Recovery delays $(\tau)$ were investigated between $10 \mathrm{~ms}$ and $20 \mathrm{~s}$. The relaxation 183 delay was set to be $>5 \mathrm{~T}_{1} . \mathrm{T}_{1} \mathrm{~s}$ were calculated from curve fitting and peak intensities which 184 were obtained from the spectra recorded for different recovery delays. Jeol, curve fitting 185 software was utilized during this process.

186 Statistical analysis

187 All data generated and collected during in vitro taste analysis by both the e-tongue and taste 188 sensing system TS-5000Z were treated by statistical methods. Results were expressed as raw 189 data in $\mathrm{mV}$ of the sample relative measurement to the reference. Sensor signal results were 190 evaluated via multivariate data analysis. Multivariate analysis, such as principal component 191 analysis (PCA), was used to reduce the multidimensional space without losing information. 192 Using PCA, the most abundant information contained in the original data could be 193 transformed into the first principal component (PC-1), and the second most abundant 194 information is transformed into the second component (PC-2). For multivariate data analysis, 195 raw data were pretreated by mean centring and scaling to unit variance. Data processing, 196 graphical illustration and statistical interpretation of the results were carried out using Excel 1972010 (Microsoft, Redmond, WA, USA). INSENT and Alpha MOS software. 


\section{Results and discussion}

\section{Hot-melt extrusion process: Particle morphology and size distribution}

202 Extrusion processing of all PRP based formulations was performed at $155^{\circ} \mathrm{C}$ with relatively

203 lower screw speed of $15 \mathrm{rpm}$ in order to allow homogenous blending of the drug/polymer

204 binary mixtures. The rationale underlying selecting high processing temperature was due to

205 the higher Tgs of polymeric carriers used. Various formulation trials were conducted at the 206 optimization stage ranging a drug loading 10-20\% (w/w ratios). But keeping the final dose in 207 the finished product e.g. tablets in account, $10 \%(\mathrm{w} / \mathrm{w})$ drug loading was chosen to proceed 208 with. Preliminary results showed no significant differences in terms of the solid state of the 209 extrudates and physical performance between the formulation containing $20 \%$ PRP and 10\% 210 PRP. Another reason underlying the selection of PRP- a cationic charged substance, as a 211 model drug and two different polymers (anionic charged) as carriers, was to possibly 212 facilitate an intermolecular interactions in order to mask unpleasant taste of the bitter API.

213 Theoretical miscibility parameter calculations showed that the solubility parameter of PRP 214 (21.94 $\left.\mathrm{MPa}^{1 / 2}\right)$ calculated by Van Krevelen equation is quite close to that of L100 (22.75 $\left.215 \mathrm{MPa}^{1 / 2}\right)$ and Acryl-EZE (21.65 $\mathrm{MPa}^{1 / 2}$ ). ${ }^{[6]}$ It has been reported in previous studies that if the 216 difference of the solubility parameters between drug and polymer is less than $7 \mathrm{MPa}^{1 / 2}$, then 217 the polymer is likely to be miscible with the API to form an amorphous solid dispersions. ${ }^{[6]}$

218 As a result the cationic PRP may interact with the functional groups of the negatively charged 219 polymers to effectively mask bitter taste of the drug.

220 SEM was used to examine the surface morphology of the drug and extrudates. The 221 extrudates containing L100 and Acryl-EZE showed homogenous particles distribution on the 222 extrudates surface with PRP (Fig. 1) indicating excellent HME processing of the extruded 223 materials to form solid dispersions. The particle size distribution depicted in Fig. 1 shows 224 particle sizes lower than $500 \mu \mathrm{m}$ for most formulations ranging from $40-400 \mu \mathrm{m}$. A small 225 percentage can be seen at sizes $<40 \mu \mathrm{m}$ as the milling process was optimized to reduce fines 226 in the final extruded batches.

\section{Solid state analysis}

228 DSC was conducted in order to analyze the solid state (crystalline or amorphous) of the pure 229 drug, polymers, drug/polymers binary mixtures and drug/polymer extrudates. The thermal 230 transition of PRP in Fig. 2 showed an endothermic peak corresponding to its melting point at $231166.65^{\circ} \mathrm{C}(\Delta \mathrm{H}=-126.25 \mathrm{~J} / \mathrm{g})$. The bulk polymers showed $\mathrm{Tgs}$ at $83.97^{\circ} \mathrm{C}$ and $164.83^{\circ} \mathrm{C}$ 232 corresponding to Acryl-EZE and L100, respectively (Fig. 2). A sharp melting peak was also 233 observed in the Acryl-EZE thermogram at $59.2^{\circ} \mathrm{C}$ (data not shown), corresponds to the 
234 presence of crystalline plasticizers in the co-processed formulation. ${ }^{[19]}$ MTDSC analysis of 235 the binary physical blends of PRP/L100 and PRP/Acryl-EZE exhibited PRP endothermic 236 peaks shifted at slightly lower temperatures of $162.41{ }^{\circ} \mathrm{C}$ to $153.62^{\circ} \mathrm{C}$ indicating a 237 drug/polymer interaction at small extent. The same shift at lower temperatures was also 238 observed for the Tgs of the polymers at $73.16^{\circ} \mathrm{C}$ and $98.82{ }^{\circ} \mathrm{C}$ for PRP/Acryl- EZE and 239 PRP/L100, respectively.

Furthermore, the extruded PRP/Acryl-EZE (and L100) extrudates exhibited single glass transition peaks at 63.36 and $74.84^{\circ} \mathrm{C}$, respectively which indicates the presence of drug/polymers miscibility and formation of molecular solid dispersions. When the two components are miscible the $\mathrm{Tg}$ of the extruded sample lies between the Tgs of the individual components (amorphous drug and polymers) according to Gordon - Taylor equation. ${ }^{[6,19]}$ The Tg of PRP was determined at $34.74^{\circ} \mathrm{C}$ (data not shown). DSC analysis confirmed the presence of molecular dispersions in all extruded formulations.

Previous studies ${ }^{[6]}$ showed that the diffraction patterns of both PRP physical mixtures exhibited crystalline peaks with reduced intensities corresponding to pure drug. The diffractograms of the extruded formulations were characterized with the absence of drug intensity peaks indicating amorphous or molecularly dispersed state.

\section{In vivo taste masking}

The masking efficiency of the developed granules was evaluated in vivo (approved by University of Greenwich, UK ethics committee) with the assistance of six healthy human volunteers (age $18-25$ ). The statistical data collected from the in vivo study for the pure active substance and the extruded formulations are depicted in Fig. 1. The data analysis showed significant suppression $(\mathrm{p}<0.05)$ of the bitter taste for the API. These results demonstrate the influence of the polymeric carriers and importance of drug loading in the final formulation. Both polymers showed effective taste masking capacity with descending order L100> Acryl-EZE. Furthermore, the HME formulations presented excellent masking effect for active concentrations (10\%) of the API. This could be due to the possible drug polymer interactions in the solid dispersions manufactured during extrusion process. In the solid dispersions cationic active substance (PRP) may have interacted with the functional group of the negatively charged polymers. These interactions facilitated a hydrogen bonding interaction between the active amide group of API and carboxylic group of polymers and consequently masked the bitter taste of the active. A similar study has also been reported elsewhere. ${ }^{[2]}$ In Fig. 3 the sensory data obtained from the panelists interestingly showed that the taste masking efficiency of L100 is not similar to that of Acryl-EZE for the API used. This could be attributed to the $\mathrm{pH}$ dependant dissolution properties of Acryl-EZE ( $\mathrm{pH} \geq 5.5$ ) 
269 compared to that of $\mathrm{L} 100(\mathrm{pH} \geq 6)$ as the saliva represents a basic $\mathrm{pH}(\sim 7.4)$ in healthy

270 individuals. However, the sensory scores of the API in different formulations are within the

271 range (below 2) which has been demonstrated as optimum by in vitro evaluations.

272 In vitro taste evaluations (Astree e-tongue)

273 Astree e-tongue was used for in vitro taste analysis of the drug and active formulations.

274 Principal Component Analysis (PCA) associated to complementary data processing was used.

$27{ }^{[20]}$ Based on the statistical analysis taste maps were constructed in order to determine the

276 distances between active and polymer solutions. Actually the distance between each active

277 formulation and its corresponding placebo is indicative of how close or how far the taste of

278 the two samples is. The interpretation of the taste maps suggests that the shorter the distance

279 (Euclidean distance) between active and placebo (polymer), the better the taste masking of

280 the active ingredient. Thus the distance between any drug polymer pairs in the taste maps is

281 indicative of the taste masking efficiency of the extruded polymer formulations from which

282 the estimated Discrimination Index (DI in \%) can be determined for each solution. This

283 indicator (DI) takes into account the average difference between the pairs (i.e drug and active

284 formulation or polymer and active formulation) to compare the dispersion or taste masking

285 effect. It is assumed that the higher the DI values (maximum 100\%), the longer the distance

286 between groups and the lower the masking effects.

287 In Fig. 4a, the taste map shows significant discrimination between placebo and active

288 solutions with PRP. Liquid sensors were able to detect the presence of the drug in the

289 extruded formulations. Considering the pure drug in deionized water the extrudates with

290 L100 (10\% drug loading $\mathrm{w} / \mathrm{w})$ shows a better taste improvement compared to that of Acryl-

291 EZE (Fig. 4a). The distance between the placebo and the active formulations indicates the

292 efficiency of the taste masking of the active by both polymers. The observed distance

293 proximity between extrudates of PRP and placebo is noticeable (for an example, 19\% taste

294 improvements of PRP with L100). This trend is likely to be linked with a pH influence of

295 Acryl-EZE in deionized water ( $\mathrm{pH} \sim 5.5)$ which leads to a higher separation of placebo from

296 the active formulations by dissolving faster than $\mathrm{L} 100(\mathrm{pH}>6.0)$. From the PCA graphs it

297 can be seen that the placebo, the API, and the extrudates are discriminated which means

298 significant taste differences.

299 Based on the Astree e-tongue experimental results it was also possible to design the

300 DI graphs for the drug - polymer combinations. In Fig. 4b, it can be seen that the distance

301 between active and placebo formulations with Acryl-EZE (DI 62\%) is higher than that of 
302 L100 (DI 40\%) in the extrudates, indicating better taste masking efficiencies of L100 (19\% 303 taste masking/improvement) than Acryl-EZE polymeric system.

304 However, this was expected as the use of deionized water was intentionally selected 305 in order to test the sensitivity of the Astree e-tongue in variations of the drug dissolution 306 rates. As mentioned above the polymers dissolve in different $\mathrm{pH}$ which results faster drug 307 release for Acryl-EZE compared to L100.

308 Sensory correlated models based on Partial Least Square (PLS) were built to evaluate 309 the correlation with sensory scores. The correlation model is considered as valid and fits with panel perception $\left(\mathrm{R}^{2}>0.80\right)$. But it should be taken with care as all data on sensory tests

311 (number of panelists, variability on measurement) were not communicated. It's quite obvious

312 from the Fig. 4c that the in vitro taste assessment studies carried out with Astree e-tongue

313 correlated very well with the in vivo panelists data $\left(\mathrm{R}^{2}=0.9892\right.$ (Acryl-EZE); 0.9959 314 (L100)).

315 PRP was found to be quite bitter by the panelists (sensory score 5) similar to the 316 Astree e-tongue evaluation. The impact of Acryl-EZE carrier was negligible as Acryl-EZE 317 itself was also found not to be bitter (sensory score 1). Contrary, PRP/L100 formulations 318 demonstrated improved taste masking even though the bulk polymer showed threshold319 moderate bitterness. However, the PLS were in good agreement by complementing the in 320 vivo study, where the panelists recorded a moderate taste with L100 (and no taste with Acryl321 EZE).

A further statistical analysis was performed by considering the standard deviations 323 (SD) and therefore the relative standard deviations (RSD) of all extruded formulations. The 324 findings of the standard deviations studies for bulk drugs and the extrudates are summarized 325 in Table 2. It can be seen that the statistical analysis of SD and RSD for all formulations, 326 showed positive results towards effective taste masking of bitter PRP. It is accepted that the 327 scale of interpretation measure is $\mathrm{SD}<50$ : Fair and $\mathrm{SD}<30$ : good, respectively. Based on 328 this scale of interpretation, the results showed (Table 2) that the calculated mean SD values 329 for all formulations is $\leq 13$ which suggests good taste masking of the API in the extruded 330 formulations.

\section{INSENT TS-5000Z sensing system}

333 The in vitro masking effect of the extruded formulations in artificial saliva was also evaluated 334 by using the INSENT TS-5000Z e-tongue. The distance percentages (\%) between active 335 substances and formulation solutions were estimated in four different time intervals ( $0.5 \mathrm{~min}$, 
$3361 \mathrm{~min}, 10 \mathrm{~min}$ and $30 \mathrm{~min}$ ) as they are indicative of taste masking efficiency of the extruded

337 formulations. In addition, the discrimination index (DI, \%) was determined for each solution.

338 Initial trials showed that the BT0 sensor of TS-5000Z system responded to the DPD and PRP

339 at the each concentration ranging from 0.03, 0.1, 0.3 and $1 \mathrm{mM}$. Therefore, BT0 sensor can

340 be useful for detecting bitterness of the API in the concentration ranging from 0.03 to $1 \mathrm{mM}$.

341 In contrast with the Astree e-tongue, in INSENT TS-5000Z system, the lower DI

342 values the longer the distance in taste responses between the pairs (drug and formulations)

343 and thus a higher discrimination, which means greater masking effect. The DI (\%) values can

344 help to assess the significance of difference between the formulations. In Fig. 5a the bitter

345 taste suppression of PRP in the L100 extrudates is quite significant even after $30 \mathrm{~min}$ as the

346 DI index (\%) is only about $60 \%$ while after $1 \mathrm{~min}$ DI is $40 \%$ (DI index (\%) close to $0 \%$

347 indicates no taste). In contrast the PRP/ Acryl-EZE extrudates (Fig. 5b) did not show taste

348 suppression similar to the L100 polymer but still the DI index (\%) estimated by the BT0

349 sensor around $98 \%$ after $30 \mathrm{~min}$ and $85 \%$ in $1 \mathrm{~min}$, respectively was considered effective (but

350 less than L100).

351 The normalized taste graphs showed significant discrimination between all active 352 formulations and active ingredient solutions (Fig. 5a-b) suggesting lower taste masking 353 efficiency of Acryl-EZE for the API compared to the taste suppression of L100.

354 As mentioned before, liquid sensors are able to detect the taste of the drug in the 355 masked formulations (up to $0.3 \mathrm{mM} \mathrm{API}$ ), therefore the in vitro taste masking results detected 356 by the BT0 sensor are quite sensitive and consistent. For that reason, in comparison with the 357 pure drug in the reference solutions (artificial saliva) the extrudates exhibited taste masking 358 effects. As mentioned above this could be attributed to the $\mathrm{pH}$ dependency of both polymers 359 which present different drug release due to their different $\mathrm{pH}$ values. However the e-tongue sensor did perceive the taste of bitter APIs from the dissolved polymer matrices.

Sensory correlated models were built to evaluate the correlation with sensory scores. The correlation model was considered as valid and fitted with panel perception (Fig 5c) and complemented the sensory findings from the panelists' scores to conclude the statement that L100 has better taste masking efficiency than Acryl-EZE. The TS-5000Z taste sensing system demonstrated different sensitivity to each sample with high correlation $\left(\mathrm{R}^{2}=0.94\right)$ to the taste scores, suggesting that the sensor responds selectively according to bitterness intensity by providing quantitative information. as shown in Fig. 5d it was not possible to detect any taste for both L100 and Acryl-EZE. 
370

371

372

373

374

375

376

377

378

379

380

381

382

383

384

385

386

387

388

389

390

391

392

393

394

395

396

397

398

399

400

401

402

403

Apparently the contribution of the bulk polymers was not taken in account as INSENT uses a different approach compared to that of Astree e-tongue.

\section{Fourier Transform Infra-Red (FT-IR) analysis}

FT-IR has been used to study interactions in drug/polymer dispersions by providing valuable information regarding the oppositely charged ionic drug/polymer interactions at molecular level. ${ }^{[23]}$ By showing the appearance of additional bands, alterations in wavenumber position or broadening of functional groups compared to the spectra of the pure drug and polymer the FTIR spectra gives an indication of drug/polymer interactions. The FTIR spectra for the extruded formulations are shown in Fig. 6.

The characteristic bands of CO- vibrations of the carboxylic acid groups in L100 and Acryl-EZE are shown at $\sim 1705 \mathrm{~cm}^{-1}$ and of the esterified carboxylic groups at $\sim 1735 \mathrm{~cm}^{-1}$. The FTIR spectra of the PRP extrudates in comparison with the pure materials are depicted in Fig. 6, which showed a new absorption band at $\sim 1560$ and $\sim 1555 \mathrm{~cm}^{-1}$ for PRP/ L100 and PRP/ Acryl-EZE, respectively. This is considered to be the result of the presence of amine group alongside the carboxyl group in the solid dispersions. ${ }^{[24]}$ During the FTIR process the resonance is possible between the two CO-bands within COO- groups. As a result, the characteristic CO- absorption is replaced by the band of auto-symmetrical vibrations of the COO- group in the $1555-1560 \mathrm{~cm}^{-1}$ region of the FTIR spectra ${ }^{[24,25]}$ which belongs to the polymer (L100 or Acryl-EZE). This type of spectra changes provides strong evidence of strong interactions between the anionic methacrylate polymers (-COO) and the cationic PRP (amine group) by enabling the formation of hydrogen bonds with the amine group of the drug.

\section{NMR analysis}

${ }^{1} \mathrm{H}_{1}$ NMR spectroscopy was employed to monitor the possible chemical changes at the molecular level by analyzing chemical shifts of NMR signals. Such a change has been observed in regards with the chemical shifts in the ${ }^{1} \mathrm{H}$ NMR spectra of drug and drug/polymer solutions. ${ }^{[26,27]}$ Previous studies showed that NMR analysis carried in DMSO successfully revealed possible drug/polymer interactions in molecular level. Initially solid state NMR was conducted in order to elucidate possible drug/polymer interactions; however the low drug loading in our formulations didn't accord NMR a meaningful interpretation.

${ }^{1} \mathrm{H} \mathrm{T}_{1}$ NMR experiments were used to analyse spin relaxation times. Different relaxation rates of nuclear spins can be related to aspects of molecular structure and additionally to internal molecular motion. The reasoning behind these experiments was to 
404 look at potential changes of the drug's molecular motion, before and after the extrusion.

405 Indeed, it would be assumed that the free drug (with a low molecular weight) would have

406 quite a high molecular motion leading to fairly high $\mathrm{T}_{1}$ relaxation delays. After formulation,

407 any consequence of an interaction between the drug and polymer would result in a decrease

408 in the amount of molecular motion observed for the drug. It can be seen in Fig. 7 that the $\mathrm{T}_{1}$

409 relaxation times have significantly been decreased in all PRP/L100 formulations. About 16-

41020 folds of decrease in the $\mathrm{T}_{1}$ relaxation times have been observed in the extruded

411 formulations. This significantly indicates that the free drug (with a low molecular weight) had

412 quite a high molecular motion leading to fairly high $\mathrm{T}_{1}$ relaxation delays (times) while the

413 extruded formulations showed very low $\mathrm{T}_{1}$ relaxation delay. This was due to the strong

414 drug/polymer interactions leading to a significant decrease in the relaxation time. $\mathrm{T}_{1}$

415 relaxation delays are particularly sensitive to intermediate molecular motions which result in

416 short $\mathrm{T}_{1} \mathrm{~s}$. Molecules which have fast or slow molecular motion can have comparable $\mathrm{T}_{1} \mathrm{~s}$. ${ }^{[6]}$

417 This NMR analysis indicates the presence of molecular interaction between the drug 418 and polymers in solutions, although the type of the interactions cannot be elucidated. In

419 addition, the presence of such intermolecular interaction can contribute to the possible taste 420 masking mechanism of all drug/polymer combinations during extrusion.

421

\section{Conclusions}

423 In this study the performance of two e-tongues was evaluated for the development of taste 424 masked PRP formulations processed by hot melt extrusion. The optimized formulations were 425 also evaluated in vivo by panellists and showed very good masking efficiency. Both e426 tongues confirmed that the extruded formulations of PRP/L100 demonstrated better taste 427 masking compared to those of PRP/ Acryl-EZE. However, each e-tongue interpreted different 428 extent of taste masking efficiency. The e-tongues evaluation suggests that results should be 429 cautiously considered in comparison to panellist's scores. The NMR and FT-IR analysis 430 confirmed possible drug/polymer intermolecular interaction which could explain the 431 mechanism underlying the taste suppression in all extruded formulations.

\section{Acknowledgements}

433 The authors would like to thank Attila Aranyos, Marion Bonnefille (Alpha MOS, France) and 434 Dr Massaki Habara and Teraoka Makoto (INSENT, Japan) for their support to run the in vitro 435 taste assessment studies.

\section{Conflict of interest}

437 The authors report no conflicts of interest. 
438

439

440

441

442

443

444

445

446

447

448

449

450

451

452

453

454

455

456

457

458

459

460

461

462

463

464

465

466

467

468

469

470

471

472

\section{Abbreviations}

Active pharmaceutical ingredient, API; Differential Scanning Calorimetry, DSC; Dispersion Index, DI; Eudragit L100, L100; European Medicines Agency, EMA; Fourier Transform Infra-Red, FT-IR; Food and Drug Administration, FDA; glass transition, Tg; Hot melt extrusion, HME; Nuclear Magnetic Resonance, NMR; propranolol HCl, PRP; Partial Least Square, PLS; Principal Component Analysis, PCA; Relative standard deviation, RSD; Scanning Electron Microcopy, SEM; Standard deviation, SD.

\section{References}

1. Maniruzzaman $\mathrm{M}$ et al. Taste masking of paracetamol by hot-melt extrusion: an in vitro and in vivo evaluation. Eur J Pharm and Biopharm 2012; 80(2): 433-42.

2. Gryczke A et al. Development and evaluation of orally disintegrating tablets (ODTs) containing ibuprofen granules prepared by hot melt extrusion. Coll and Surface B Biointerfaces 2011; 86(2): 275-84.

3. Ley JP Masking bitter taste bymolecules, Chemosensory Perception 2008; 1:5877.

4. Mennella J, Beauchamp G.Optimizing oral medication for children, Clinical Therapeutics 2009; 30: 2120-2132.

5. Regulation (EC) No 1901/2006 of the European Parliament and of the Council of 12 December (2006) on Medicinal Products for Paediatric Use.

6. Maniruzzaman $\mathrm{M}$ et al. Drug-polymer intermolecular interactions in hot-melt extruded solid dispersions. Int J Pharm 2013; 443(1-2): 199-208.

7. Cram A. Challenges of developing palatable oral paediatric formulations, Int $\mathbf{J}$ Pharm 2009; 365: 1-3.

8. EMA,2007.(http://ec.europa.eu/enterprise/pharmaceuticals/paediatrics/docs/draft guideline pip 2007-02.pdf). Accessed 23/10/2012.

9. Abu-Diak OA et al. Understanding the performance of melt-extruded poly(ethylene oxide)-bicalutamide solid dispersions: characterisation of microstructural properties using thermal, spectroscopic and drug release methods. J Pharm Sci 2012; 101(1): 200-13

10. Wilson $\mathrm{M}$ et al. Hot-melt extrusion technology and pharmaceutical application. Therapeutic Delivery 2012; 3 (6): 787-797.

11. Maniruzzaman $\mathrm{M}$ et al. Taste masking of bitter APIs by using hot melt extrusion (HME). AAPS Journal 201113 (S2), T3273-T3273.

12. Ciosek $\mathrm{P}$ et al. The recognition of growth conditions and metabolic type of plants by a potentiometric electronic tongue. Electroanalysis 2006a; 18: 1266-1272. 
13. Ciosek P, Wróblewski W. The analysis of sensor array data with various pattern recognition techniques. Sensors and Actuators 2006b; B 114: 85-93.

14. Legin A et al. Electronic tongue for pharmaceutical analytics: quantification of tastes and masking effects. Anal Bioanal Chem 2004; 380: 36-45.

15. Ciosek P, Wróblewski W. Sensor arrays for liquid sensing-electronic tongue systems. The Analyst 2007; 132: 963-978.

16. Jańczyk $M$ et al. Electronic tongue for the detection of taste-masking microencapsulation of active pharmaceutical substances. Bioelectrochemistry 2006; 80: 94-98.

17. Woertz K. et al. Rational development of taste masked oral liquids guided by an electronic tongue. Int J Pharm 2010; 400: 114-123.

18. Woertz K. et al. A comparative study on two electronic tongues for pharmaceutical formulation development. J Pharm Biomed Anal 2011a; 55:272281.

19. Maniruzzaman M, Bonnefille M, Aranyos A, Snowden MJ, Douroumis D. An in-vivo and in-vitro taste masking evaluation of bitter melt-extruded drugs. $J$ Pharm Pharmacol 2014; 66(2):323-37.

20. Altan S et al. An Application of Serially Balanced Designs for the Study of Known Taste Samples with the $\alpha$-ASTREE ElectronicTongue. AAPS PharmSciTech 2014; In press.

21. Qiu S et al. Discrimination and Characterization of Strawberry Juice Based on Electronic Nose and Tongue: Comparison of Different Juice Processing Approaches by LDA, PLSR, RF, and SVM. J Agric Food Chem 2014;62 (27):6426-34

22. Woertz K et al. Taste sensing systems (electronic tongues) for pharmaceutical applications, Int J Pharm 2011b; 417: 256- 271.

23. Nair Ret al. Influence of various drugs on the glass transition temperature of poly(vinylpyrrolidone): a thermodynamic and spectroscopic investigation. Int $J$ Pharm 2001; 225: 83-96.

24. Lee HK et al. Propranolol-/methacrylic acid copolymer binding interaction, $J$ Pharml Sci 1991; 80:178-180.

25. Avram M, Mateescu GH. Infrared Spectroscopy: Applications in Organic Chemistry, Wiley/Interscience, New York. 1996. 
506

507

508

509

510

511

512

513

514

515

516

517

518

519

520

521

522

523

524

525

526

527

528

529

530

531

532

533

534

535

536

537

538

539

540
26. Qi S et al. Novel characterisation of phase separation in hot melt extruded solid dispersions: a thermal, microscopic and NMR relaxometry study. Pharm Res 2010; 27: 1869-83.

27. Qi $S$ et al. Characterisation of solid dispersions of paracetamol and EUDRAGIT ${ }^{\circledR}$ E prepared by hot melt extrusion using thermal, microthermal and spectroscopic analysis. Int J Pharm 2008; 354, (1-2):158-67. 
$\underline{\text { Tables }}$

542

543 Table 1: Sample preparation for taste masking analysis

\begin{tabular}{|l|l|c|c|c|c|c|}
\hline \multicolumn{1}{|c|}{ Description } & \multicolumn{1}{|c|}{ Type } & $\begin{array}{c}\text { Drug } \\
\mathbf{( \% )}\end{array}$ & $\begin{array}{c}\text { Polymer } \\
\mathbf{( \% )}\end{array}$ & $\begin{array}{c}\text { Drug } \\
\mathbf{( m g )}\end{array}$ & $\begin{array}{c}\text { Polymer } \\
\mathbf{( m g )}\end{array}$ & $\begin{array}{c}\text { Total } \\
(\mathbf{m g})\end{array}$ \\
\hline PRP & Active & 100 & 0 & 100 & 0 & 100 \\
\hline Acryl-EZE & Polymer & 0 & 90 & 0 & 900 & 900 \\
\hline PRP/ Acryl-EZE & Extrudates & 10 & 90 & 100 & 900 & 1000 \\
\hline L100 & Polymer & 0 & 90 & 0 & 900 & 900 \\
\hline PRP/L100 & Extrudates & 10 & 90 & 100 & 900 & 1000 \\
\hline
\end{tabular}

544

545

546 Table 2: Mean standard deviation (SD) and relative standard deviation (RSD) for each 547 solution for in vitro taste analysis by Astree e-tongue.

\begin{tabular}{lccc}
\hline \multicolumn{4}{c}{ PRP dissolution after 60s } \\
\hline Formulation & Mean SD & Mean RSD (\%) & Interpretations \\
PRP & 8.0 & 0.7 & Good \\
PRP/ Acryl-EZE & 12.0 & 0.9 & Good \\
PRP/L100 & 10.0 & 1.4 & Good \\
Acryl-EZE & 13.0 & 1.1 & Good \\
L100 & 8.0 & 0.8 & Good \\
AVERAGE & 13.0 & 0.7 & Good \\
\hline
\end{tabular}


Fig. 1

Fig. 2

Fig. 3

Fig. 4a

Fig. 4b

Fig. 4c

Fig. 5a

Fig. 5b

Fig. 5c

Fig. 5d

Fig. 6

Fig. 7
SEM images of PRP/polymer extrudates and particle size distribution. DSC thermal transitions of (i) PRP and polymers pure, (ii) PRP/polymer extruded formulations.

Sensory scores of all formulations by panelist $(n=6)$.

Signal comparison between active and placebo formulations with L100 and Acryl-EZE and PRP (dissolution for 60s).

Distance and discrimination comparison between signal of PRP pure and their formulations on Astree e-tongue (after 60s dissolution).

Sensory correlation model based on PLS with Astree e-tongue

Normalised DI (\%) of all drug/L100 formulations in four different time scale.

Normalised DI (\%) of all drug/ Acryl-EZE formulations in four different time scale.

Relationship between results of taste sensors and human taste scores for similar tastes. The standard deviations on the $x$ - and $y$-axes are the difference between the panelists' scores and measurement error $(n=6)$, respectively.

Sensor output of the two polymers, Acryl-EZE and L100. Maximum concentrations of polymers were set at 60 times the maximum API conc. (approx. $0.5 \mathrm{mg} / \mathrm{mL}$ x $60=30 \mathrm{mg} / \mathrm{mL}$ ).

FT-IR spectra of PRP extruded formulations.

Part, ${ }^{1} \mathrm{H}$ T1 spectra (aromatic region) for the PRP pure and taste masked formulations. 


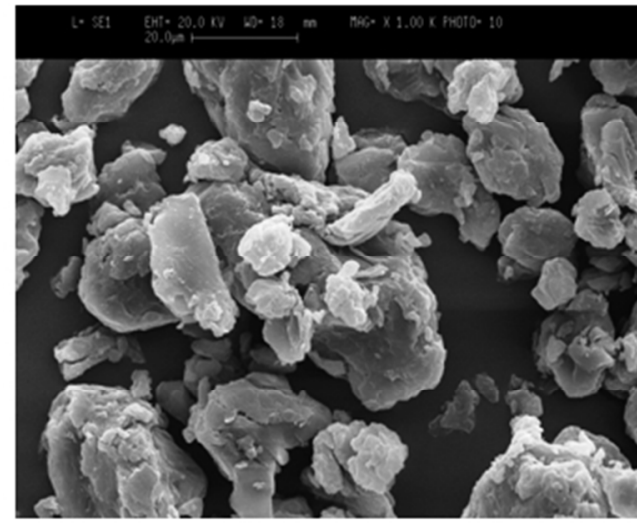

(i) PRP/L100

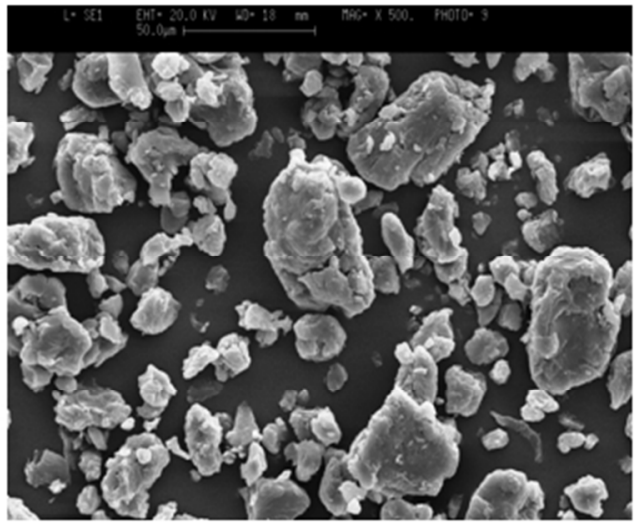

(ii) PRP/ Acryl-EZE

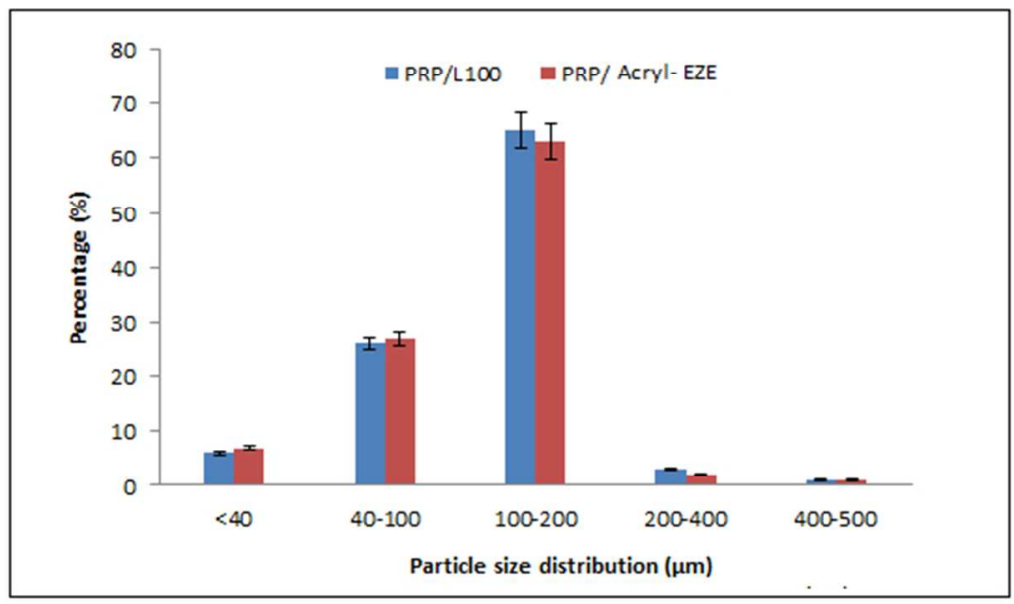

184x170mm (96 x 96 DPI) 

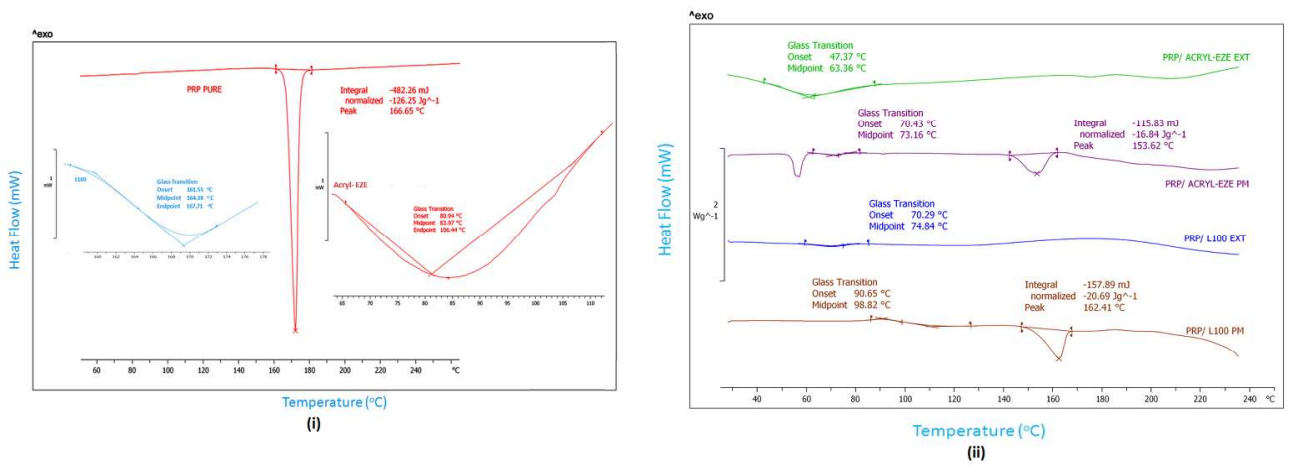

$599 \times 211 \mathrm{~mm}(96 \times 96$ DPI $)$ 


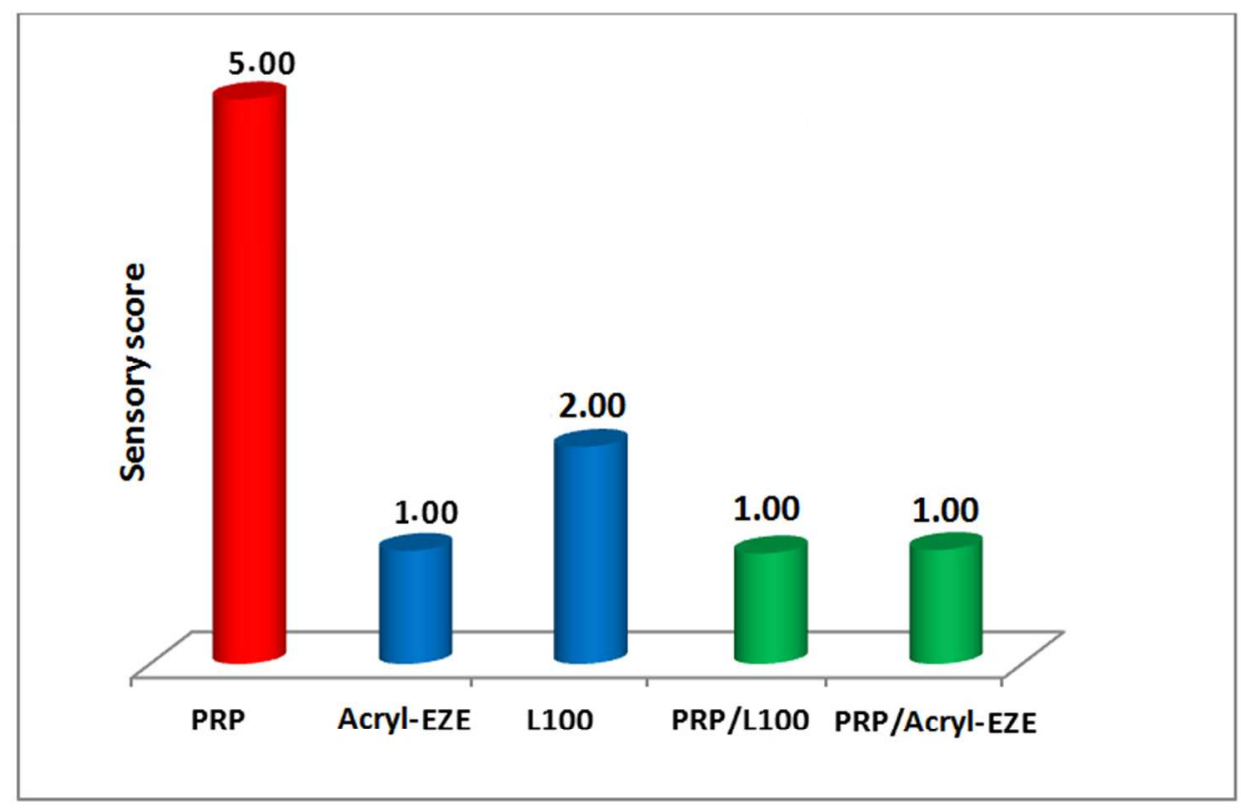

$213 \times 138 \mathrm{~mm}(96 \times 96$ DPI $)$ 
Page 23 of 31

Journal of Pharmacy and Pharmacology

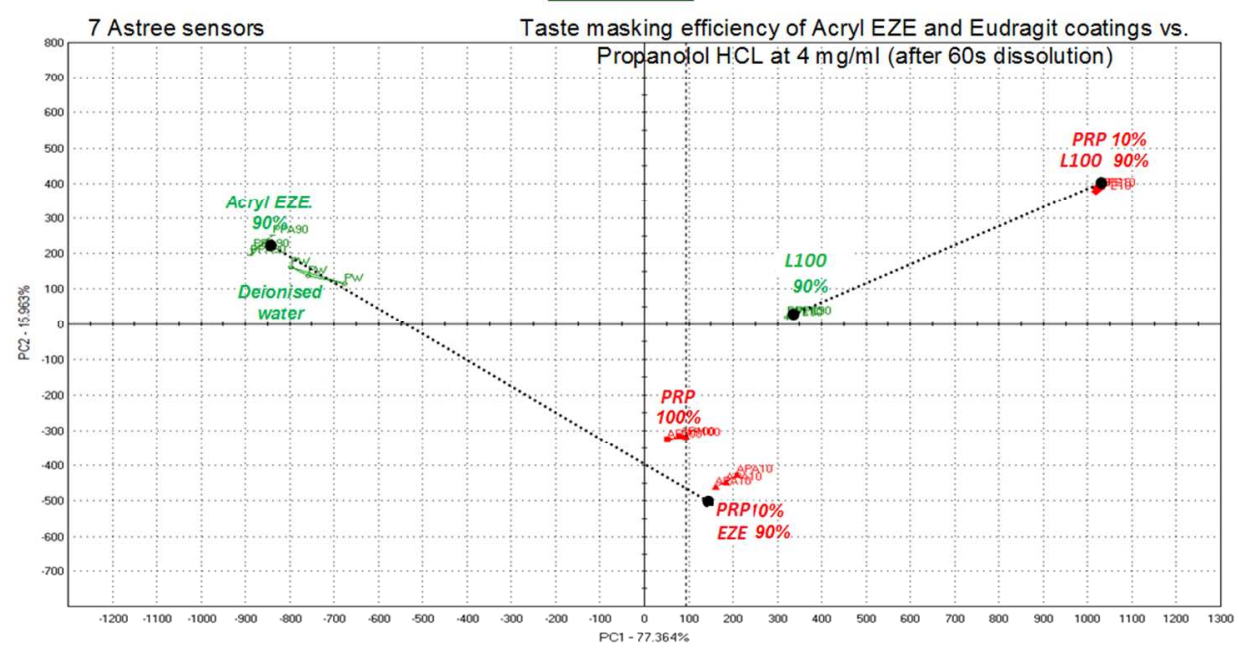

$311 \times 174 m m(96 \times 96$ DPI) 
Taste masking efficiency of coatings on Propanolol $\mathrm{HCL}(4 \mathrm{mg} / \mathrm{ml})$ after 60 s dissolution

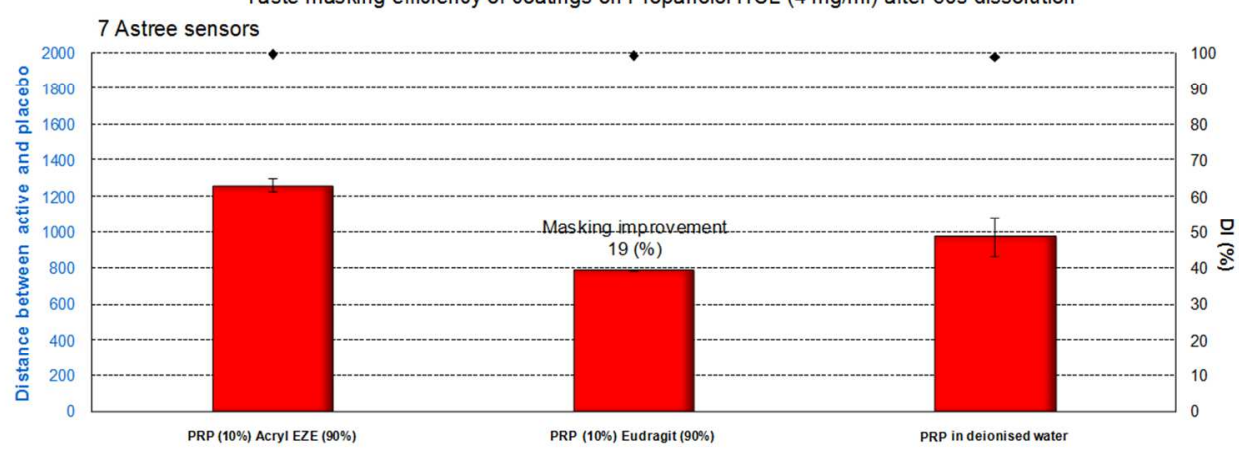

$318 \times 131 \mathrm{~mm}(96 \times 96 \mathrm{DPI})$ 
Taste correlation of polymer's formulation vs. propanolol solution (dose $100 \mathrm{mg}$ )
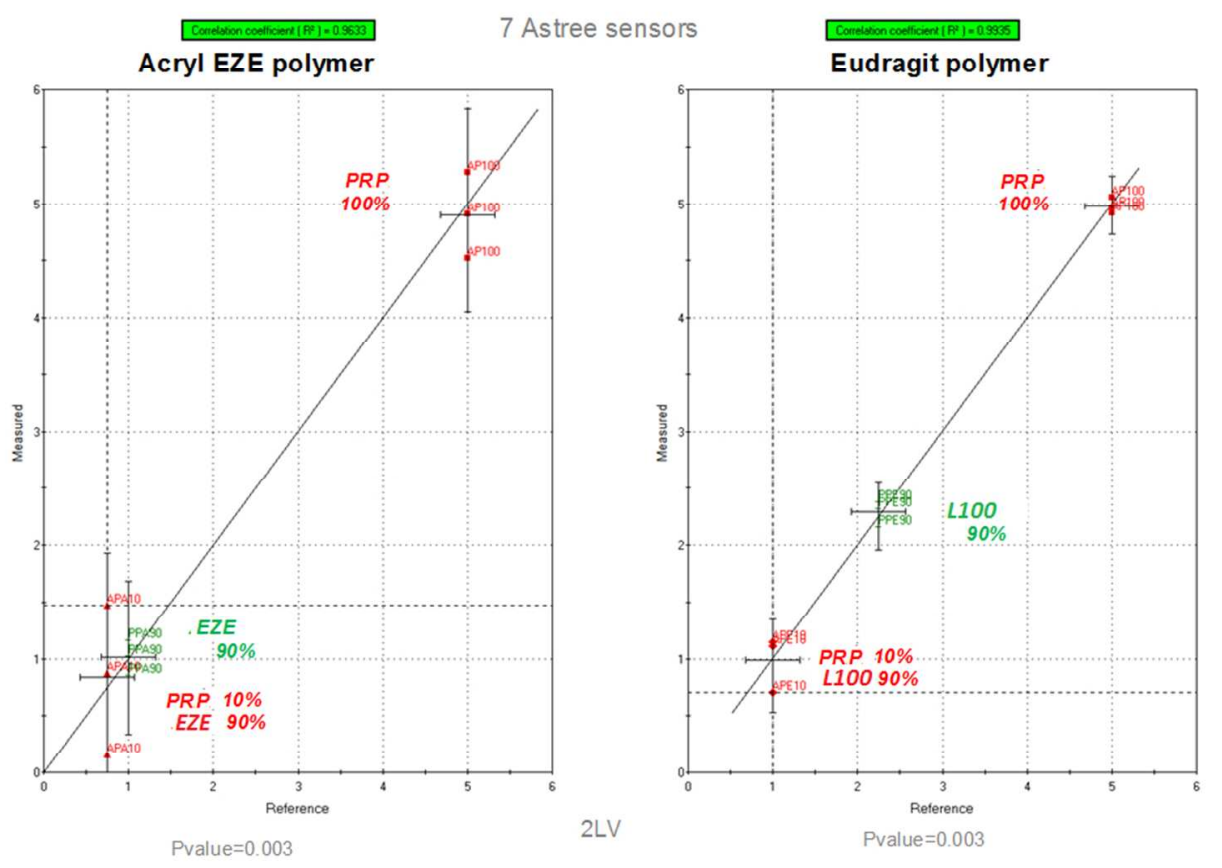

$263 \times 194 \mathrm{~mm}(96 \times 96$ DPI) 


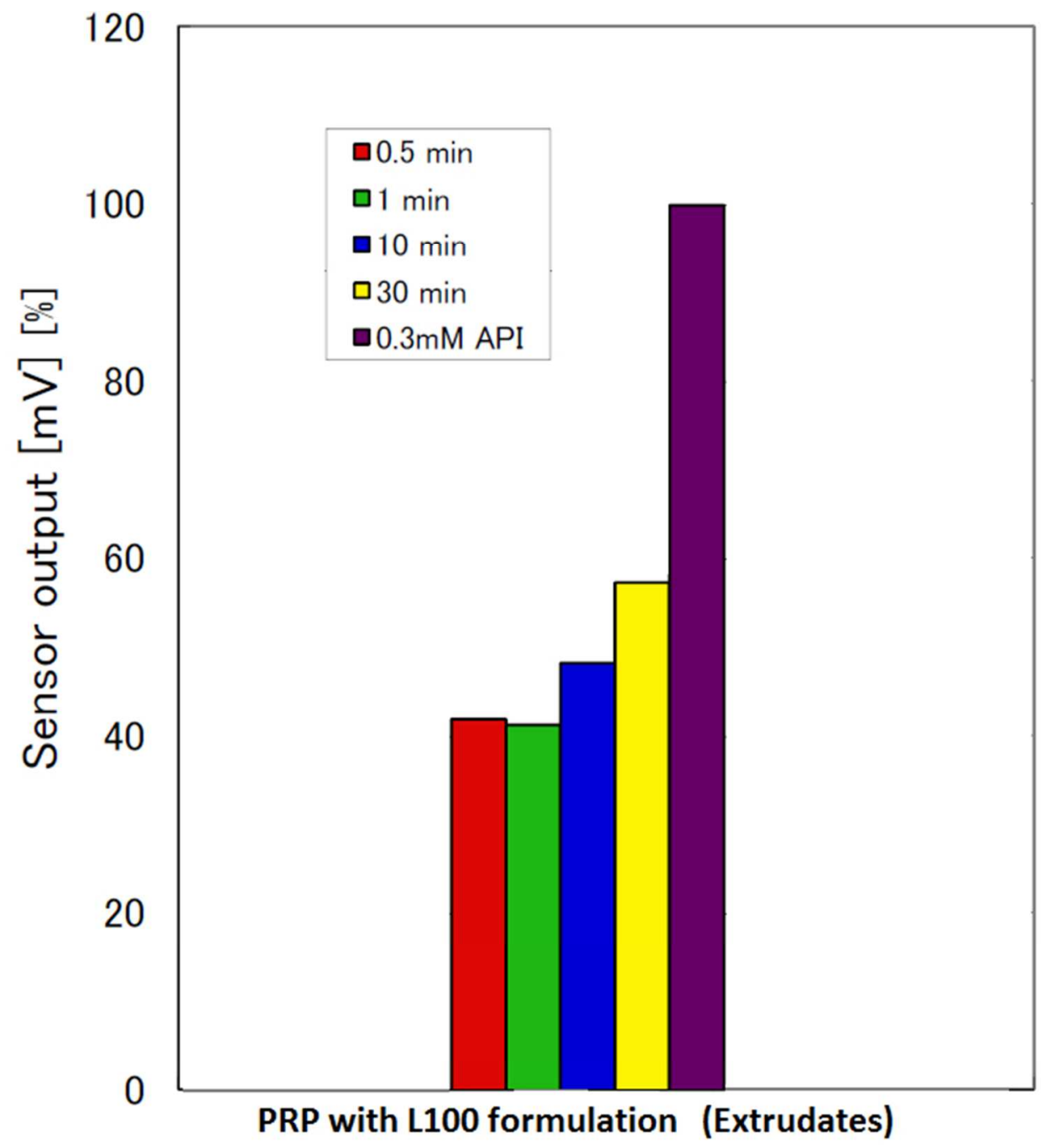

$183 \times 195 \mathrm{~mm}(96 \times 96 \mathrm{DPI})$ 


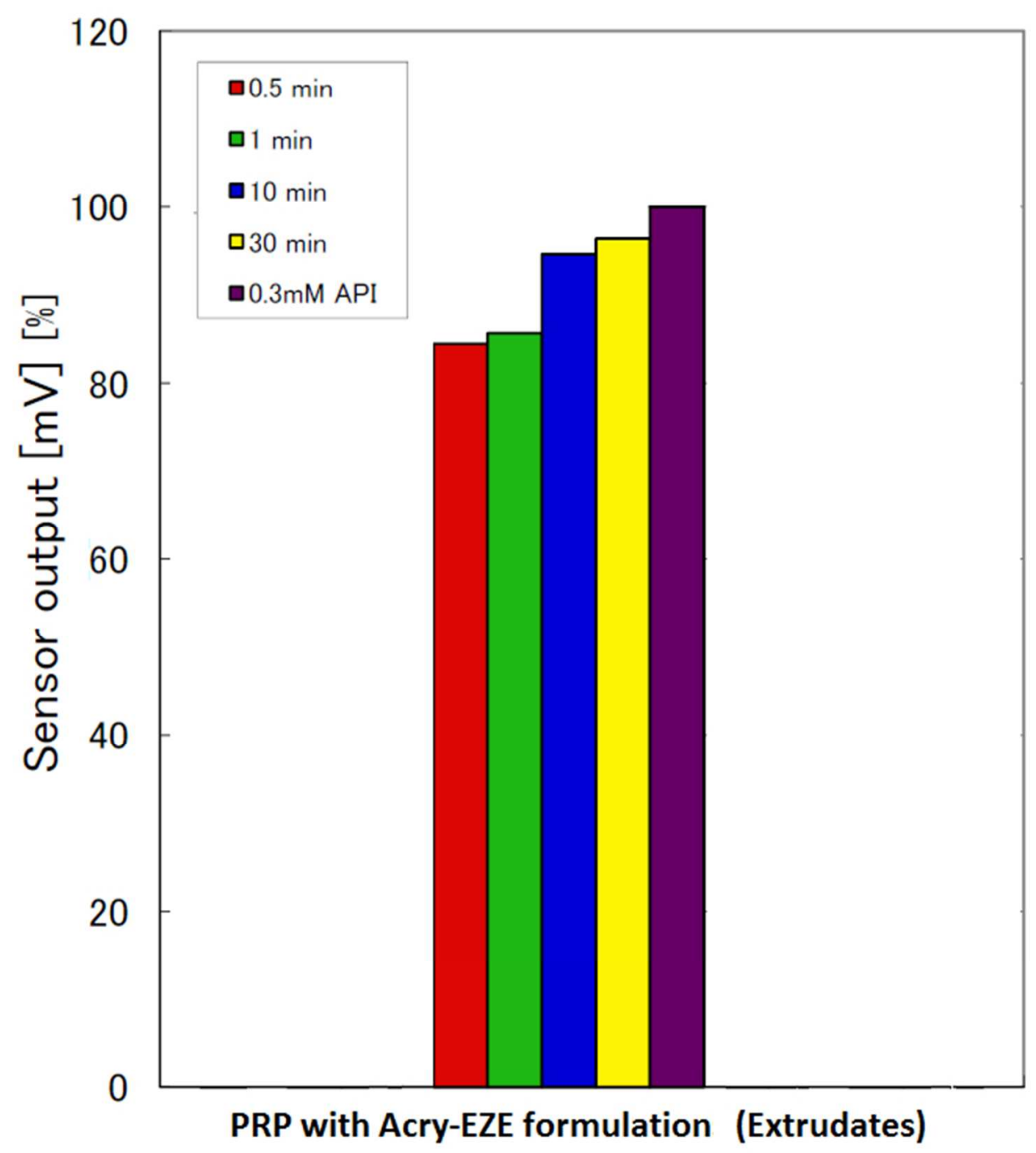

$183 \times 201 \mathrm{~mm}(96 \times 96$ DPI $)$ 


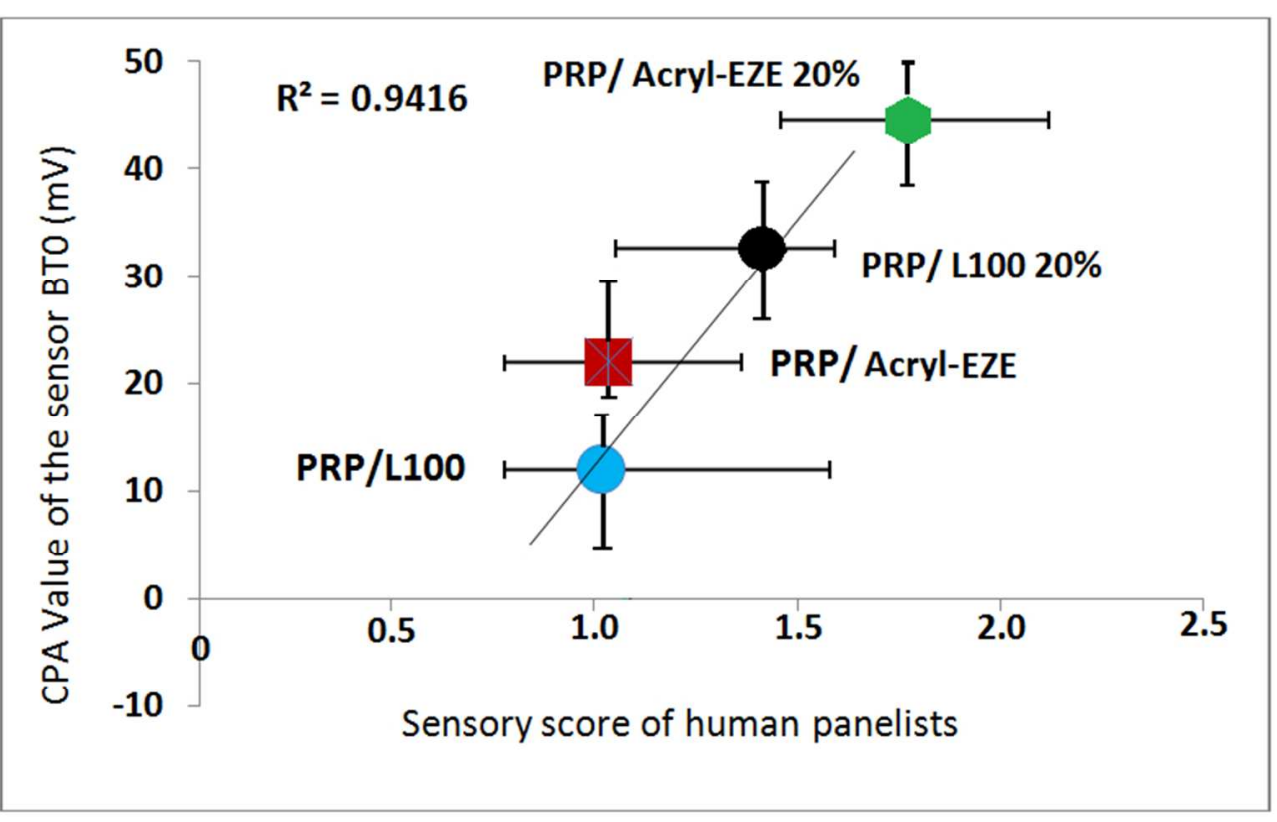

$210 \times 132 \mathrm{~mm}(96 \times 96$ DPI $)$ 


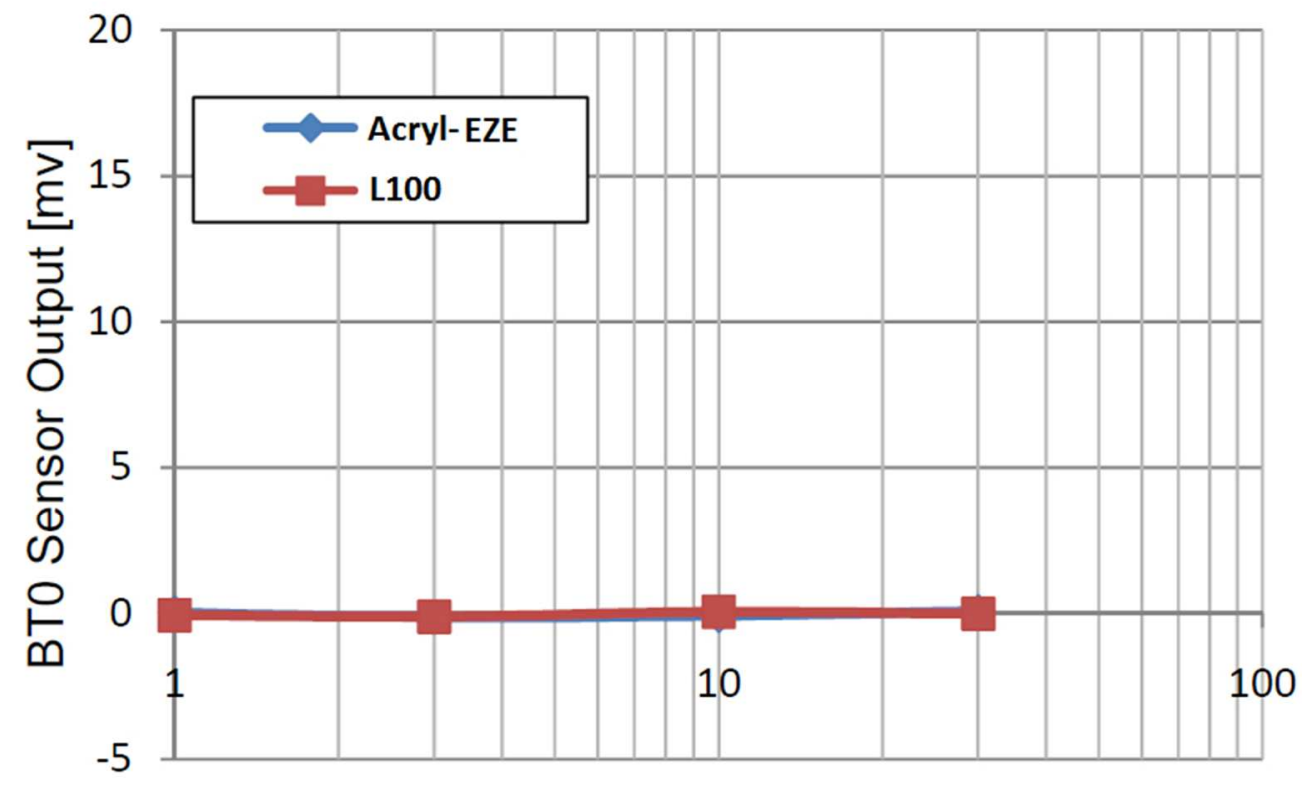

Conc. $[\mathrm{mg} / \mathrm{mL}]$

\begin{tabular}{|c|c|c|}
\hline $\mathrm{mg} / \mathrm{mL}$ & Acryl-EZE $(\mathrm{mV})$ & L100 $(\mathrm{mV})$ \\
\hline 1 & 0.01 & -0.06 \\
\hline 3 & -0.14 & -0.12 \\
\hline 10 & -0.08 & 0.05 \\
\hline 30 & 0.07 & -0.01 \\
\hline
\end{tabular}

$218 \times 203 \mathrm{~mm}(96 \times 96$ DPI) 

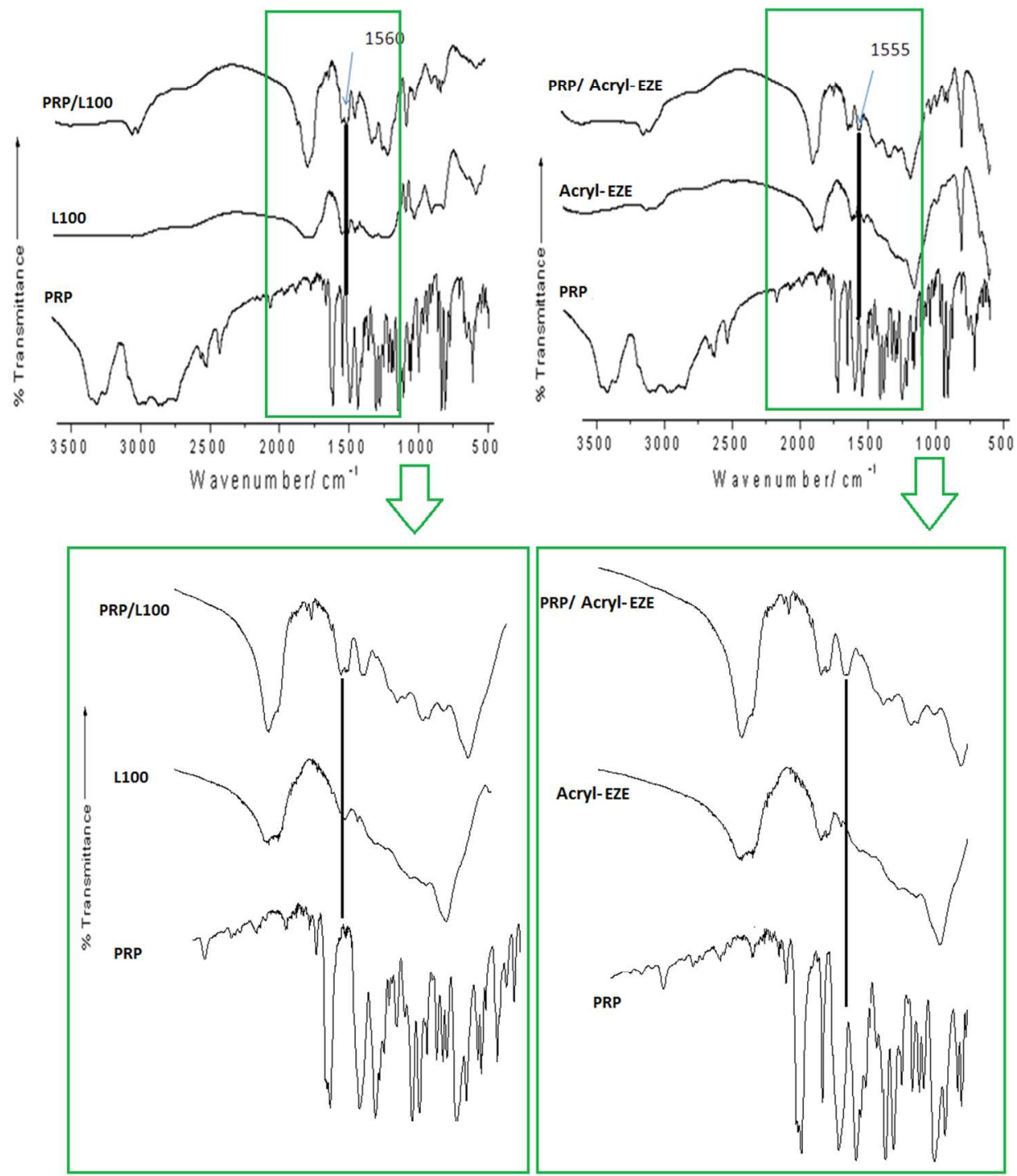

$308 \times 351 \mathrm{~mm}(96 \times 96$ DPI) 


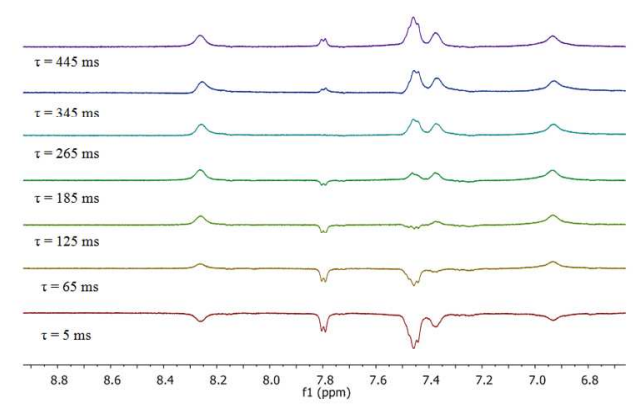

Part, ${ }^{1} \mathrm{H}$ T1 spectra (aromatic region) for the PRP/L100

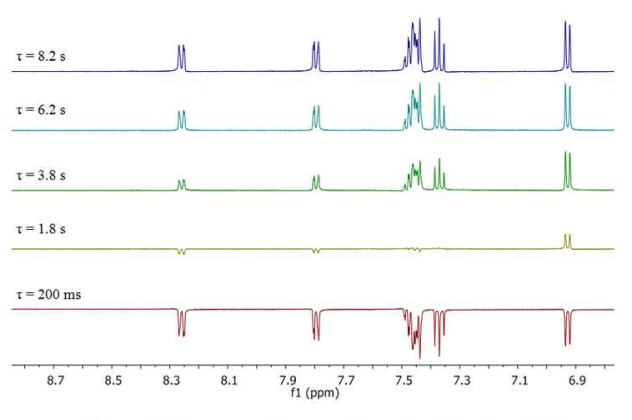

Part, ${ }^{1} \mathrm{H}$ T1 spectra (aromatic region) for the PRP

$465 \times 166 \mathrm{~mm}(96 \times 96 \mathrm{DPI})$ 